\title{
Personbezogene Faktoren im bio-psycho-sozialen Modell der WHO: Systematik der Deutschen Gesellschaft für Sozialmedizin und Prävention (DGSMP)
}

\author{
Personal Factors of the Bio-Psycho-Social Model (WHO): A Revised \\ Classification by the German Society for Social Medicine and \\ Prevention (DGSMP)
}

Autoren

Sabine Grotkamp1, Wolfgang Cibis², Silke Brüggemann ${ }^{*}$, M. Michaela Coenen ${ }^{4}$, Hans-Peter Gmünder ${ }^{*}$, Klaus Keller ${ }^{6^{*}}$, Elisabeth Nüchtern ${ }^{7^{*}}$, Urban Schwegler ${ }^{{ }^{*}}$, Wolfgang Seger ${ }^{{ }^{*}}$, Stefan Staubli ${ }^{10^{*}}$, B. Bia von Raison ${ }^{11^{*}}$, R Weißmann ${ }^{12^{*}}$, A. Bahemann ${ }^{13^{* *}}$, H. Fuchs ${ }^{14^{* *}}$, M. Rink ${ }^{15^{* *}}$, Marcus Schian ${ }^{16^{* *}}$, Klaus Schmitt ${ }^{17^{* *}}$

Institute

1 MDK Niedersachsen, Vizepräsidentin der DGSMP und Leiterin der AG „ICF“, Leiterin der Sozialmedizinischen Expertengruppe „Leistungsbeurteilung/Teilhabe“ der MDK Gemeinschaft, Hannover

2 Stellvertretender Leiter der AG „ICF“ der DGSMP

3 DRV Bund, Geschäftsbereich Sozialmedizin und Rehabilitation, Leiterin Bereich Sozialmedizin, Berlin

4 Institut für Medizinische Informationsverarbeitung, Biometrie und Epidemiologie - IBE, Lehrstuhl für Public Health und Versorgungsforschung, Pettenkofer School of Public Health, Leitung Fachbereich Biopsychosoziale Gesundheit, Ludwig-Maximilians-Universität (LMU), München

5 Schweizer Paraplegiker-Zentrum Nottwil, Universität Zürich, Direktor Schweizer Paraplegiker-Zentrum Nottwil, Schweiz

6 Rehabilitationsklinik Herzogsägmühle, Leiter Bereich Rehabilitation, Peiting-Herzogsägmühle

7 Mitglied der AG „ICF“ der DGSMP

8 Schweizer Paraplegiker-Forschung Nottwil, Gruppenleiter „Partizipation, Integration und soziale Epidemiologie“, Lehrbeauftragter der Universität Luzern, Schweiz

9 Vorsitzender des Ärztlichen Sachverständigenrates der Bundesarbeitsgemeinschaft für Rehabilitation (BAR), Hannover

10 Schweizer Paraplegiker-Zentrum Nottwil Leiter Soziale und Berufliche Integration, Schweiz

11 Mitarbeiterin des Kreises Segeberg (Jugendhilfe), Bad Segeberg

12 Katholische Universität Eichstätt Ingolstadt, Wissenschaftliche Mitarbeiterin an der Professur für psychologische Diagnostik und Interventionspsychologie, Eichstätt

13 Mitglied der AG „ICF“ der DGSMP, Nürnberg

Mitglieder haben sich in gleicher Weise eingebracht, alphabetische Nennung

** Nennung in alphabetischer Reihenfolge
14 Honorarprofessor im Fachbereich Sozial- und Kulturwissenschaften der Hochschule Düsseldorf, Düsseldorf

15 Vizepräsidentin der Deutschen Rheuma Liga und stellvertretende Vorsitzende der BAG - Selbsthilfe, Berlin

16 Teamleiter Reha- und Teilhaberecht, Fachbereich Programme und Produkte der Bundesarbeitsgemeinschaft für Rehabilitation (BAR), Frankfurt

17 Leiter Akutmedizin, Schweizer Paraplegiker-Zentrum Nottwil, Schweiz

\section{Schlüsselwörter}

Internationale Klassifikation der Funktionsfähigkeit, Behinderung und Gesundheit (ICF) - Kontextfaktoren Personbezogene Faktoren - Deutsche Gesellschaft für Sozialmedizin und Prävention (DGSMP) - Systematik

Key words

International Classification of Functioning, Disability, and Health (ICF) - contextual factors - personal factors German Society for Social Medicine and Prevention (DGSMP) - classification

Bibliografie

DOI https://doi.org/10.1055/a-1011-3161

Online-Publikation: 22.10.2019

Gesundheitswesen 2020; 82: 107-116

(c) Georg Thieme Verlag KG Stuttgart · New York

ISSN 0941-3790

\section{Korrespondenzadresse}

Dr. med. Sabine Grotkamp MHM

Leiterin der SEG 1 der MDK-Gemeinschaft

MDK Niedersachsen

Hildesheimer Straße 202

30519 Hannover

sabine.grotkamp@mdkn.de 


\section{ZUSAMMENFASSUNC}

Hintergrund Die Arbeitsgruppe „ICF“ des Fachbereichs II „Praktische Sozialmedizin und Rehabilitation“ der Deutschen Gesellschaft für Sozialmedizin und Prävention (DGSMP) entwarf 2010 eine Liste personbezogener Faktoren (PF) für den deutschen Sprachraum. Zwischenzeitlich haben die ICF und das bio-psycho-soziale Modell der WHO eine verstärkte Verankerung im SGB IX gefunden. So hat der Gesetzgeber verbindlich bestimmt, dass im Reha-Prozess eine Bedarfsfeststellung „umfassend" zu erfolgen hat. Damit sind die PF ein obligatorischer Bestandteil der Bedarfsermittlung.

Ziel Ziel der vorliegenden Arbeit ist es, eine aktualisierte Fassung der PF für die (sozialmedizinische) Begutachtung im deutschen Sprachraum vorzulegen. Dazu erfolgte eine Auseinandersetzung mit bereits publizierten Vorschlägen anderer Autoren, insbesondere mit der Arbeit von Geyh et al.

Methodik Die multiprofessionell zusammengesetzte Arbeitsgruppe überprüfte mittels einer qualitativen Herangehensweise die Grundstruktur, Konsistenz und Faktorenauswahl der DGSMP-Systematik und modifizierte diese, sofern sinnvoll und erforderlich. Die Grundsätze für die Faktorenauswahl wurden analog zur Publikation von 2010 beibehalten.

Ergebnisse Eine grundlegende Anpassung war nicht erforderlich, die Grundstruktur der Systematik blieb im Wesentlichen erhalten. Einige Kategorien wurden ergänzt, gestrichen, zusammengefasst, verschoben sowie redaktionell oder inhaltlich überarbeitet. Eine Systematik zu PF ist als solche datenschutzrechtlich grundsätzlich unbedenklich. Die Zulässigkeit einer Erhebung von einzelnen PF im Rahmen der Bedarfsermittlung und -feststellung wird nach ihrer Erforderlichkeit im konkreten Einzelfall für die Erfüllung der gesetzlichen Aufgaben beurteilt.

Ausblick Die überarbeitete DGSMP-Systematik ermöglicht es dem Anwender, relevante Einflüsse aus dem Lebenshintergrund einer Person strukturiert zu beschreiben und zu dokumentieren. Damit können Einflüsse auf die Teilhabe eines Menschen auf Grundlage des bio-psycho-sozialen Modells umfassend und transparent dargestellt werden. Der Weg zu einer justiziablen Leistungsallokation und umfassenden Nutzung, insbesondere im gesamten Reha-Prozess wird erleichtert.

\section{ABSTRACT}

Background In 2010, the ICF working group of Faculty II "Applied Social Medicine and Rehabilitation" of the German Society for Social Medicine and Prevention, DGSMP proposed a classification of personal factors (PF) for the German-speaking area. Meanwhile, the International Classification of Functioning, Disability and Health (ICF) and WHO's bio-psycho-social model were increasingly integrated into the German Social Code (Book IX for Rehabilitation and Participation). It was a legislative decision that the needs assessment for the rehabilitation process must be "comprehensive".

Aim This publication aims to present an updated classification of PF to support the socio-medical assessment. For this purpose other published papers proposing a classification of $\mathrm{PF}$ were analyzed, especially the publication of Geyh et al.

Methods The multiprofessional working group re-examined the basic structure, consistency and selection of factors of the 2010 classification using a qualitative approach and modified them if meaningful and necessary. The principles for the selection of factors were the same as in the 2010 publication (comprehensive, manageable, universal, impartial, relevant, unambiguous, focusing on finality, not regarding causality and non-discriminatory).

Results A fundamental revision was not necessary; the basic structure remained primarily unchanged. Some items were included, excluded, summarized, shifted and editorially or content-related altered. Legal expertise shows that the classification of PF and their individual use for the socio-medical assessment, if necessary for the individual rehabilitation allocation, incur no problems with regard to data-protection regulations.

Perspectives The revised classification is ready to support users to describe and document relevant influences of the life background of individuals in a structured manner. Thus, influences on functioning and participation can be described comprehensively and transparently based on the bio-psycho-social model. A justiciable allocation of benefits for persons with disabilities is facilitated.

\section{Einleitung}

Mit der Umsetzung des Übereinkommens über die Rechte von Menschen mit Behinderungen (UN-BRK) hat das bio-psycho-soziale Modell und die darauf basierende Internationale Klassifikation der Funktionsfähigkeit, Behinderung und Gesundheit (ICF) der Weltgesundheitsorganisation (WHO) im deutschen Sprachraum an Bedeutung gewonnen [1]. Ein Beispiel dafür ist das deutsche Bundesteilhabegesetzes (BTHG) im Jahr 2016. Es revidierte das Neunte Buch Sozialgesetzbuch (SGB IX) und definierte Behinderung neu. In §2 Abs. 1 Satz 1 SGB IX heißt es:

„Menschen mit Behinderungen sind Menschen, die körperliche, seelische, geistige oder Sinnesbeeinträchtigungen haben, die sie in Wechselwirkung mit einstellungs- und umweltbedingten Barrieren an der gleichberechtigten Teilhabe an der Gesellschaft mit hoher Wahrscheinlichkeit länger als sechs Monate hindern können“.

Ein weiteres Beispiel ist die in $\S \S 13$ und 19 SGB IX im Rehabilitationsrecht verankerte „individuelle und funktionsbezogene“ Ermittlung von Rehabilitationsbedarfen. Für den Bereich der Eingliederungshilfe ist die ICF als Maßstab der Instrumente der Bedarfsermittlung benannt (§118 Abs. 1 SGB IX).

\section{Mehr Autonomie für Leistungsberechtigte bedeutet: Kontextfaktoren werden wichtiger}

Mit dem SGB IX schafft der Gesetzgeber die Voraussetzungen für ein hohes Maß an Autonomie der Leistungsberechtigten in ihrer Lebensgestaltung. Die Interessen und Bedürfnisse der Leistungsberechtigen sind handlungsleitend, Beurteilungen der Leistungs- 
erbringer sowie die sich daraus ableitenden Empfehlungen und deren Umsetzung müssen mit ihm abgestimmt werden.

Diese Zielsetzung zu realisieren, bedeutet für alle Personen im Gesundheitsbereich, sich dezidiert mit den Kontextfaktoren auseinanderzusetzen, sofern sie als Förderfaktor oder Barriere für die Funktionsfähigkeit des betroffenen Menschen relevant sind. Kontextfaktoren können sowohl als Umweltfaktoren als auch von der Person selbst ausgehend als PF mit der Funktionsfähigkeit interagieren.

Zu einer bio-psycho-sozialen Perspektive auf Funktionsfähigkeit, Behinderung und Gesundheit gehört die Berücksichtigung von positiven wie negativen Wechselwirkungen (Förderfaktoren und Barrieren) zwischen dem Gesundheitsproblem, seinen Auswirkungen auf die Funktionsfähigkeit sowie den modulierenden Kontextfaktoren. Nur so können die notwendigen Maßnahmen individuell umfassend ermittelt, passgenau geplant, durchgeführt und evaluiert werden.

Mithilfe der ICF lassen sich die individuellen Auswirkungen eines Gesundheitsproblems auf die Funktionsfähigkeit systematisch und standardisiert beschreiben und dokumentieren. Während die Umweltfaktoren in der ICF klar konzeptualisiert und klassifiziert sind, stellt die WHO für die PF nur eine stark vereinfachte konzeptionelle Definition zur Verfügung: „Personbezogene Faktoren sind der spezielle Hintergrund des Lebens und der Lebensführung eines Menschen und umfassen Gegebenheiten des Menschen, die nicht Teil ihres Gesundheitsproblems oder -zustands sind“ und hinterlegt diese mit einigen Beispielen wie Geschlecht, ethnische Zugehörigkeit, Alter, Fitness, Lebensstil, Gewohnheiten, Bewältigungsstile [1]. Die Beurteilung dieser Faktoren sei, falls notwendig, den Anwendern überlassen.

\section{Relevanz personbezogener Faktoren}

Die Haltung der WHO, PF nicht zu klassifizieren, erschwert die Verständigung in einer gemeinsamen, von Anwendungsfeldern und Disziplinen/Professionen unabhängigen Sprache im Sozial- und Gesundheitswesen sowie die Vergleichbarkeit medizinischer Evaluation und der darauf basierenden Berichte und Entscheidungen. Eine allgemein anerkannte Systematik der PF bietet nicht nur ein gemeinsames Arbeitswerkzeug für die Anwender, sondern auch einen Standard, der von allen in die Bedarfsermittlung involvierten Personen bei Entscheidungen berücksichtigt werden kann. Dies begünstigt die Interraterreliabilität von Entscheidungen und trägt zur Vergleichbarkeit im Entscheidungsprozess bei [2]. PF sind zudem ein zentraler Bestandteil einer ganzheitlichen Betrachtung der Situation von Menschen mit Behinderungen [1]. Faktoren wie Selbstvertrauen oder Ernährungsgewohnheiten stellen nicht nur wesentliche Determinanten von Gesundheit und Funktionsfähigkeit dar, sondern sind als Ansatzpunkte für Interventionen der Rehabilitation und Integration systematisch zu berücksichtigen [3]. Eine systematische Beschreibung von relevanten PF erhöht die Transparenz und Nachvollziehbarkeit der im Rahmen der Bedarfs- und Leistungsermittlung gefällten Entscheidungen und sichert eine personenzentrierte Rehabilitations- und Integrationsstrategie. Die explizite statt implizite Dokumentation personbezogener Förderfaktoren und Barrieren für die Funktionsfähigkeit steigert zudem die Verständlichkeit medizinischer Gutachten und gibt den Betroffenen die Möglichkeit, bei als ungerechtfertigt empfundenen Aussagen und Entscheidungen zu intervenieren [4]. Dies liefert ein zentrales ethisch-moralisches Argument für die strukturierte Berücksichti- gung von PF bei der Bedarfsermittlung und Durchführung von Teilhabeleistungen.

\section{Systematisierung personbezogener Faktoren}

Aufgrund einer aufkommenden Unzufriedenheit mit der unzureichenden Konzeptualisierung und fehlenden Systematisierung der $\mathrm{PF}$ in der ICF haben sich in den vergangenen Jahren verschiedene Arbeitsgruppen mit der Kategorisierung von PF beschäftigt [3, 5-12]. Jedoch beschränken sich die meisten dieser Vorschläge entweder auf spezifische Anwendungsbereiche (z. B. Arbeitsreintegration) oder bestimmte Teilaspekte der PF (z. B. Lifestyles oder subjektive Erfahrungen). Der einzige Vorschlag, der einen umfassenden Blick auf PF erlaubt, ist jener von Geyh et al. [3]. Auch die DGSMP stellte sich der Herausforderung, eine Systematik zu PF für den deutschen Sprachraum zu entwickeln. Der Entwurf wurde 2010 in einer ersten Fassung, 2012 mit Beispielen unterlegt [13, 14] und für eine internationale Diskussion in englischer Sprache [15] publiziert. Die DGSMP-Systematik wurde in Deutschland wie international intensiv diskutiert, so auch auf der ICF-Anwenderkonferenz im Rahmen einer WHO Tagung im April 2018 in Hamburg.

Als Grundlage für die sozialmedizinische Begutachtung von Rehabilitations- und Teilhabeleistungen sowie für eine umfassende Betrachtung im Rahmen des gesamten Reha-Prozesses hat sie Eingang in zahlreiche Schriften der Bundesarbeitsgemeinschaft für Rehabilitation (BAR) gefunden [16]. Bei der Erarbeitung des vom Bundesministerium für Arbeit und Soziales in Auftrag gegebenen Basiskonzepts für die Bedarfsermittlung in der beruflichen Rehabilitation [17] wurde diese Systematik für die Zuordnung der Instrumente zur ICF-Komponente PF verwendet. Daneben findet sie in wissenschaftliche Arbeiten Berücksichtigung, so bei Geyh et al. [3], Rapp [18] und Mahdi [19].

Ziel der vorliegenden Arbeit ist eine Aktualisierung der DGSMPSystematik zu PF aus 2010 auf der Grundlage einer diskursiven Auseinandersetzung mit Vorschlägen anderer Autoren, insbesondere von Geyh et al. [3] sowie den bis dato vorliegenden Rückmeldungen.

\section{Methodik}

Die 2010 publizierte DGSMP-Systematik zu PF bildete die Grundlage für die Überarbeitung durch eine multiprofessionelle deutschschweizerische Arbeitsgruppe der DGSMP.

\section{Beteiligte}

Beteiligt waren mit der ICF vertraute Personen von Leistungsträgern und -erbringern, der Wissenschaft und Selbsthilfe unterschiedlicher Professionen und Funktionen: Sozialmedizin, Rehabilitationsmedizin, Gesundheits- und Rehabilitationswissenschaft, Rehabilitationsmanagement, berufliche Integration, Rechtswissenschaft, Physio-/Ergotherapie, Sozialpädagogik, Psychologie, Gesundheitsökonomie, Politikberatung und Verwaltungswirtschaft.

\section{Grundlagen für die Überarbeitung}

Es bestand Konsens, die Konzeptualisierung personbezogener Faktoren als meist überdauernde Merkmale einer Person beizubehalten. Ebenso sollte die Struktur der Systematik nach Möglichkeit gleichbleiben, damit Anwender in Studien und Formularen zur Bedarfsermittlung bereits erfasste Daten weiterhin nutzen können. 
Dabei waren Spezifität und Praktikabilität für die Ermittlung von Teilhabebedarfen in Einklang zu bringen.

Die Grundsätze für die Faktorenauswahl (umfassend, universell, wertneutral, handhabbar, relevant, eindeutig, final ausgerichtet und nicht diskriminierend) galten analog zur Erstpublikation [13].

Zusätzlich wurden die folgenden Quellen für die Überarbeitung der Systematik verwendet:

1. Fachspezifische Rückmeldungen:

- aus den ICF-Anwenderkonferenzen seit 2010

- der ICF Plattform und Anwendertagungen der Swiss Association of Rehabilitation (SAR)

- im Fachaustausch mit anderen Fachgesellschaften

- im Rahmen des b3-Bedarfsermittlungskonzeptes

- aus dem ärztlichen Sachverständigenrat der BAR

- von Anwendern aus dem Bereich der sozialmedizinischen Begutachtung

2. Bisher publizierte Ansätze zur Systematisierung personbezogener Faktoren [3, 5-12]

Die genannten neun Ansätze zur Systematisierung von PF wurden im Hinblick auf evtl. fehlende Kategorien in der DGSMP-Systematik analysiert. Im Ergebnis war nur der Ansatz von Geyh et al. für die weitere Diskussion von Bedeutung, da dieser als einziger umfassend angelegt ist [3]. Ein Co-Autor der Publikation konnte für die Überarbeitung der DGSMP-Systematik gewonnen werden.

Geyh et al. entwickelten ihre Systematik zu PF in einem mehrstufigen Ansatz. Neben der Sichtung von wissenschaftlicher Literatur und strukturierten Diskussionsrunden wurden Sekundäranalysen von qualitativen Daten aus der Entwicklung von ICF Core Sets unter Zuhilfenahme der Inhaltsanalyse nach Schreier [20] verwendet. Im Ergebnis entstand eine Systematik von PF, die sich in drei Teile mit sieben Bereichen unterteilt. Die Struktur der Systematik nach Geyh et al. findet sich in > Tab. 1; die Struktur der DGSMPSystematik von 2010 in $>$ Tab. 2.

\section{Vorgehen}

Die Überarbeitung der Systematik erfolgte in acht strukturierten und moderierten 1- bis 2-tägigen Sitzungen der Arbeitsgruppe von Oktober 2017 bis Juli 2019. In den Sitzungen wurden Arbeitsaufgaben auf die Mitglieder der Arbeitsgruppe entsprechend deren Tätigkeitsschwerpunkt und Expertise verteilt und zum darauffolgenden Treffen zur Kommentierung in die Gruppe zurückgegeben. Bestand innerhalb der Gruppe keine ausreichende Expertise für bestimmte Themen, wurden externe Experten konsultiert. In den Sitzungen erfolgte die Diskussion und ggf. Spezifizierung sowie Revision der vorgelegten Beiträge im Konsensverfahren. Auch die Ergebnisse der Konsultationen wurden in der Arbeitsgruppe diskutiert und ggf. modifiziert.

\section{Ergebnisse}

Im Folgenden ist die Kurzversion der Systematik (2. Ebene Kategorien) mit ihren fünf Kapiteln dargestellt.

Die Langversion der Systematik mit Definitionen, Beispielen sowie Ein- und Ausschlusskriterien kann von der DGSMP-Homepage heruntergeladen werden (https://www.dgsmp.de/).

\section{Kapitel 1}

\section{Allgemeine Merkmale einer Person}

Dieses Kapitel befasst sich mit vorgegebenen allgemeinen Merkmalen einer Person wie Alter und Geschlecht, die sich als Barriere oder Förderfaktor auf die Funktionsfähigkeit auswirken können. Das Kapitel umfasst nicht Merkmale, die einer Gesundheitsstörung oder Krankheit entsprechen.

Alter (i110-119)

Entwicklungsstand einer Person unter kalendarischen, psychosozialen und biologischen Aspekten

i110 Kalendarisches Alter

i112 Psychosoziales Alter

i114 Biologisches Alter

i118 Alter, anders bezeichnet

i119 Alter, nicht näher bezeichnet

Geschlecht (i120-129)

Die Zugehörigkeit zu einem Geschlecht (z. B. männlich, weiblich, divers) in Bezug auf das biologische Geschlecht sowie die geschlechtliche Identität (erlebtes und gelebtes geschlechtliches Selbst).

i120 Biologisches Geschlecht

i122 Soziales Geschlecht (Gender)

i128 Geschlecht, anders bezeichnet

i129 Geschlecht, nicht näher bezeichnet

i198 Allgemeine Merkmale einer Person, anders bezeichnet

i199 Allgemeine Merkmale, nicht näher bezeichnet

\section{Kapitel 2}

\section{Physische Faktoren}

Das Kapitel befasst sich mit der angeborenen oder erworbenen körperlichen Konstitution wie Faktoren des Körperbaus und anderen physischen Faktoren, die die Funktionsfähigkeit und ihr Veränderungspotenzial beeinflussen können. Körperliche Potenziale/Talente sind eingeschlossen.

Es betrifft nicht Schädigungen von Körperfunktionen -strukturen.

i210 Körpermaße, Körperform und Körperzusammensetzung

i220 Bewegungsbezogene Faktoren

i221 Faktoren des Herz-, Kreislauf- und Atmungssystems

i222 Faktoren des Stoffwechsels

i223 Faktoren der Sinnesorgane

i298 Physische Faktoren, anders bezeichnet

i299 Physische Faktoren, nicht näher bezeichnet

\section{Kapitel 3}

\section{Mentale Faktoren}

Das Kapitel befasst sich mit den grundlegenden Faktoren der Persönlichkeit sowie kognitiven und mnestischen Faktoren, die als Förderfaktoren und Barrieren die Funktionsfähigkeit beeinflussen können. Enthalten sind Intelligenz und angeborene mentale Talente. Es betrifft nicht Schädigungen mentaler Funktionen. Haben die 
Merkmale Krankheitswert, werden sie nicht als PF klassifiziert, sondern bei den mentalen Funktionen.

Faktoren der Persönlichkeit (i310-349)

Hierbei handelt es sich um mentale Faktoren, die das Naturell einer Person betreffen, individuell auf Situationen zu reagieren, einschließlich der psychischen Charakteristika, die eine Person von einer anderen unterscheiden.

i310 Extraversion

i315 Faktoren der Emotionalität

i320 Gewissenhaftigkeit

i325 Offenheit gegenüber neuen Erfahrungen

i330 Umgänglichkeit

i335 Selbstvertrauen

i340 Optimismus

i348 Faktoren der Persönlichkeit, anders bezeichnet

i349 Faktoren der Persönlichkeit, nicht näher bezeichnet

Kognitive und mnestische Faktoren (i350-369)

Hierbei handelt es sich um mentale Faktoren, die die Intelligenz, Kognition und Informationsverarbeitung betreffen und für die Funktionsfähigkeit von Relevanz sein können.

i350 Faktoren der Intelligenz

i355 Kognitive Faktoren

i360 Mnestische Faktoren

i368 Kognitive und mnestische Faktoren, anders bezeichnet

i369 Kognitive und mnestische Faktoren, nicht näher bezeichnet

i398 Mentale Faktoren, anders bezeichnet

i399 Mentale Faktoren, nicht näher bezeichnet

Kapitel 4

Einstellungen, Handlungskompetenz und Gewohnheiten

Das Kapitel befasst sich mit persönlichen Einstellungen, Kompetenzen und Gewohnheiten, die als Förderfaktoren und Barrieren die Funktionsfähigkeit beeinflussen und von großer Bedeutung für die Motivation sein können. Es befasst sich nicht mit Aktivitäten, die infolge eines Gesundheitsproblems beeinträchtigt sind.

Einstellungen (i410-429)

Einstellungen haben in unterschiedlichem Umfang emotionale, kognitive und motivationale Anteile, die das eigene Handeln und Leben in bestimmten Bereichen betreffen und sich auf meist überdauernde Überzeugungen, Sichtweisen und Wertvorstellungen beziehen. Die emotionalen Anteile betreffen Gefühle und emotionale Bewertungen. Kognitive Anteile reflektieren Anschauungen, Erwartungen, Erklärungs- und Attributionsansätze sowie rationale Bewertungen. Motivationale Anteile beziehen sich auf Interessen, Präferenzen und persönliche Ziele. Einstellungen können die Sicht auf die Welt und deren Deutung betreffen, die generelle Zuschreibung von Ursachen zu Ereignissen, die Rolle des Einzelnen in der Gesellschaft oder seine Sicht auf die Gesellschaft. i410

i411

i413

i416

i418

i419

i420

i421

i422

i425

i428

i429

Handlungskompetenz (i430-449)

Persönliche Kompetenzen können für die Bewältigung der Auswirkungen von Krankheit und Behinderung relevant sein. Zur Handlungskompetenz gehören Kenntnisse, Fähigkeiten und Fertigkeiten, die Entwicklung von Handlungsstrategien, die Planung von Handlungen und deren zielgerichtete Ausführung sowie die dazugehörige Handlungsbereitschaft und die Reflexion über das eigene Tun.

In diesem Teilkapitel (i430-i449) werden Faktoren subsumiert, die eine Grundlage für eine entsprechende Handlung bilden, nicht jedoch das Handeln selbst.

\section{i430 Sozialkompetenz \\ i431 Sprachkompetenz \\ i433 Methodenkompetenz \\ i436 Selbstkompetenz (Empowerment) \\ i438 Fachkompetenz \\ i442 Medienkompetenz \\ i448 Handlungskompetenz, anders bezeichnet \\ i449 Handlungskompetenz, nicht näher bezeichnet}

Gewohnheiten (i450-479)

Durch Wiederholung selbstverständlich gewordene Verhaltensmuster.

i450 Ernährungsgewohnheiten

i453 Gewohnheiten beim Konsum von Genussmitteln

i456 Bewegungsgewohnheiten

i459 Gewohnheiten in alltäglichen Routinen

i460 Freizeitgewohnheiten

i462 Sexualgewohnheiten

i465 Kommunikationsgewohnheiten

i468 Hygienegewohnheiten

i471 Gewohnheiten im Umgang mit Geld und materiellen Gütern

i478 Verhaltensgewohnheiten, anders bezeichnet

i479 Verhaltensgewohnheiten, nicht näher bezeichnet

i498 Einstellungen, Handlungskompetenz und Gewohnheiten, anders bezeichnet

i499 Einstellungen, Handlungskompetenz und Gewohnheiten, nicht näher bezeichnet 


\section{Kapitel 5} Lebenslage

Das Kapitel befasst sich mit dem Status einer Person in ihrem unmittelbaren und weiteren Umfeld. Dieser kann die Funktionsfähigkeit und ihr Veränderungspotenzial beeinflussen.

\section{i510 Familiärer Status}

i513 Status im unmittelbaren und weiteren sozialen Kontext

i515 Wohnsituation

i520 Beschäftigungsstatus

i525 Wirtschaftlicher Status

i527 Rechtlicher Status

i530 Gesellschaftlicher Status

i535 Kultureller Status

i540 Zugehörigkeit zu ethnischen Gruppen

i550 Bildungsstatus

i598 Lebenslage, anders bezeichnet

i599 Lebenslage, nicht näher bezeichnet

Eine Übersicht zur Struktur DGSMP-Systematik von 2019 ist in Tab. 3 enthalten.

\section{Änderungen zum Vorentwurf und Erläuterungen Kapitel 1 und 2}

\section{- Genetische Faktoren}

Die Kategorie „Genetische Faktoren“ wurde in der überarbeiteten Systematik nicht mehr aufgeführt, da fast jede Ausprägung eines Menschen (Körperstrukturen/-funktionen, PF) auf der Basis von genetischen Faktoren betrachtet werden kann.

\section{- Auflösung der 3. Ebene}

In beiden Kapiteln wurde im Hinblick auf die Einheitlichkeit der Gliederung die 3. Ebene neu bewertet und in die zweite Ebene eingeordnet $>$ Tab. 4.

\section{Kapitel 3}

\section{- Verortung von Talent und Begabung - Das komplexe Konstrukt Intelligenz}

Die überarbeitete Fassung der Einführungssätze in Kapitel 2 „Physische Faktoren“ und Kapitel 3 „Mentale Faktoren“ orientiert sich am Modell von Deiglmayr, Schalk \& Stern [20] . Der Begriff Begabung beschreibt das Potenzial eines Menschen in einem spezifischen Leistungsbereich. Darunter fallen einerseits kognitive Begabungen, z. B. die mathematische und sprachliche Begabung, andererseits auch soziale, motorische sowie künstlerische Begabungen. Eine Begabung kann vorliegen, auch wenn diese nicht umgesetzt oder angewandt wird.

Intelligenz ist ein multimodaler, aus Elementen des Verstehens, des Abstrahierens, des Schlussfolgerns, der Problemlösung und der Situationsbewältigung bestehender Faktor. Der Verbleib der Kategorie „Faktoren der Intelligenz“ wurde kontrovers diskutiert, da es sich einerseits um ein komplexes Konstrukt aus kognitiven und mnestischen Anteilen handelt, andererseits aber auch Talent (z. B. musische Begabung) enthalten sind. Aufgrund seiner praktischen Relevanz verblieb die Katego- rie in der Systematik. Ebenso wurden für eine differenzierte Beschreibung die spezifische Kategorie „Kognitive und mnestische Faktoren“ (i 350-369) in der Systematik belassen.

Um in Abgrenzung zur Intelligenz die realisierte Begabung in einem nicht-kognitiven Bereich zu beschreiben, wird der Talentbegriff verwendet. Genauso wie die Intelligenz entwickelt sich das Talent aus dem Zusammenspiel von Begabung und Person-Umwelt-Interaktion.

\section{- Faktoren der Persönlichkeit}

Mit der Umbenennung des Items „Zuverlässigkeit“ (i320) in „Gewissenhaftigkeit“ orientiert sich die Systematik an empirisch begründeten psychologischen Theorien [21]. Zuverlässigkeit bildet neben u. a. Sorgfalt, Genauigkeit und Prinzipientreue lediglich einen Teilaspekt von Gewissenhaftigkeit ab, der als übergeordneter Faktor zu betrachten ist.

\section{Kapitel 4}

\section{- Einstellungen}

Die Struktur des Teilkapitels „Einstellungen“ blieb größtenteils erhalten. Im Gegensatz zum Ansatz von Geyh et al., der differenziert Gefühle, Gedanken/Überzeugungen und Motive unterscheidet, subsumiert der DGSMP-Ansatz diese Bereiche im Teilkapitel „Einstellungen“, da sie sowohl emotionale, kognitive als auch motivationale Anteile enthalten. Die den Einstellungen zugrundeliegenden spezifischen Anteile könnten in einer weiteren Entwicklung der Systematik auf der dritten Ebene weiter differenziert werden.

- Die komplexen Konstrukte Motivation und Selbstwirksamkeit

Motivation wird auch weiterhin nicht als eigenständige Kategorie aufgeführt, auch wenn sie in vielen Fragestellungen von großer Bedeutung ist, da es sich nicht um ein eigenständiges und trennscharfes Konstrukt handelt. Motivation beinhaltet emotionale Faktoren, die zur Verfügung stehende Energie, Antrieb, Willen und Handlungsmotive. Begriffe wie Beweggründe, Handlungsbereitschaft, Bedürfnis, Wirksamkeitsstreben, Selbstmanagement, Streben, Drang, Wünsche, Triebkraft, Begierde, Ziele, Motive, Motivierung, Wille, Selbstbestimmung, Bereitwilligkeit werden oft mit dem Begriff Motivation verbunden. Zudem wird in diversen Modellen zwischen extrinsischer und intrinsischer Motivation bzw. Quellen der Motivation unterschieden.

Die der Motivation zugrundeliegenden Einzelitems finden sich bei den mentalen Faktoren und den Einstellungen. Einstellungen bspw., die unzweifelhaft in das Gesamtkonzept Motivation einfließen, können spezifiziert wesentlich differenzierter als Förderfaktoren oder Barrieren wahrgenommen und Wechselwirkungen zwischen den einzelnen Faktoren dargestellt werden.

Die Frage, ob ein Mensch gewillt, bereit oder mental in der Lage ist, eine Rehabilitation durchzuführen, ist unter Einbezug des Betroffenen vom Sachverständigen zu beurteilen. Die ggf. zusammenfassende Erkenntnis in einer bestimmten Situation „Reha-Motivation nicht gegeben“ darf nicht als PF missverstanden werden.

Vergleichbar komplex ist das Konstrukt der Selbstwirksamkeit, das in verschiedenen Bereichen der Rehabilitation (z. B. der beruflichen Integration) eine zentrale Rolle spielt. Selbstwirksamkeitsüberzeugungen, d. h. die Überzeugung gewisse 
Handlungen ausführen und Ziele erreichen zu können, sind primär kognitiver Natur und können sich im Rahmen von traumatischen Ereignissen (z. B. Querschnittlähmung) verändern. In der DGSMP-Systematik sind sie daher im Teilkapitel „Einstellungen“ verortet. Gleichzeitig ist Selbstwirksamkeit aber auch geprägt von eher überdauernden Merkmalen (z. B. hohes Selbstvertrauen) und kann im Rahmen einer depressiven Symptomatik beeinträchtigt sein (z. B. Wertlosigkeitsgefühle). Insofern ist Selbstwirksamkeit ein Konstrukt mit stabilen und variablen Anteilen, das sich sowohl auf Ebene mentaler Funktionen als auch PF manifestieren kann.

\section{- Kompetenzen}

Zwischenzeitliche Diskussionen im Zusammenhang mit der deutschen Gesetzgebung (BTHG) und hierbei insbesondere der Bedarfsermittlung von Teilhabeleistungen haben die Arbeitsgruppe zu einer Schärfung des Begriffs „Kompetenzen“ bewogen.

Der 2010 gewählte Begriff der „Grundkompetenz“ als Kapitelüberschrift wurde erweitert zu „Handlungskompetenz“, da Kompetenzen nicht nur auf der Grundlage von physischen Potenzialen, kognitiven Begabungen und mentalen Talenten, sondern auch vom Wissenszuwachs in den einzelnen Entwicklungsstufen abhängen.

Ein Zusammenhang zwischen Kompetenzen und Aktivitäten besteht insofern, als Kompetenz einerseits das Ergebnis eines Lernprozesses ist, andererseits eine Grundlage für effektives und effizientes Handeln bildet. Die Abgrenzung ergibt sich aus der unterschiedlichen Perspektive: Aktivitäten fokussieren darauf, ob und in welchem Ausmaß eine bestimmte Tätigkeit im Zusammenhang mit einem Gesundheitsproblem nicht oder nur unter Schwierigkeiten ausgeführt werden kann. Kompetenz als PF bestimmt neben Umweltfaktoren und weiteren PF die Qualität des Handelns. Kompetenzen können als Förderfaktor oder als Barriere für Aktivitäten und Teilhabe wirken.

Eine Aphasie nach einem Schlaganfall würde beispielsweise zu einer Beeinträchtigung der Aktivität Sprechen (d330) führen. Die Fähigkeit, sich grammatikalisch korrekt und dem jeweiligen Kontext entsprechend auszudrücken, ist hingegen in der überabeiteten Systematik unter „Sprachkompetenz“ (i431) zu verorten. Der Beschreibung der Kategorie Sprachkompetenz liegt das Verständnis einer operationalen Sprachkompetenz zugrunde, die neben der Kenntnis über die Regeln einer Sprache auch die Fertigkeit zur praktischen Anwendung der Sprache einbezieht. Das Item Sprachliche Verständigung in der Systematik von 2010 (i545) wurde umbenannt in Sprachkompetenz und demzufolge in Kapitel 4 „Handlungskompetenz“ verortet, da die Sprachkompetenz wesentlich zur Ausbildung sämtlicher Teilkompetenzen von Handlungskompetenz, insbesondere dem Aufbau von Sach- und Sozialkompetenz, beiträgt.

\section{Kapitel 5: Lebenslage}

Als Lebenslage wird die Gesamtheit der äußeren Bedingungen bezeichnet, durch die das Leben von Personen beeinflusst wird und innerhalb derer eine Person sich entsprechend ihrem Handlungsspielraum entwickelt.

Durch Einbindung in meist mehrdimensionale soziale Bezüge, in denen sich Interessen und Handlungsziele von Personen entwickeln, ergeben sich Spielräume oder Begrenzungen für individuelle
Handlungsmöglichkeiten (Output). Diese sind in der vorgelegten Systematik als PF unter dem Begriff „Lebenslage“ zusammengefasst.

Davon abzugrenzen sind materielle und immaterielle Ressourcen wie Vermögenswerte oder familiäre Unterstützung, also Umweltfaktoren im Sinne der ICF. Sie bilden Handlungsvoraussetzungen (Input).

Während z. B. die technische Ausstattung einer Wohnung ein Umweltfaktor ist, stellt das Wie und Wo man lebt einen PF dar.

Aus den Handlungsvoraussetzungen und Handlungsmöglichkeiten der Lebenslage resultieren individuelle Einstellungen (Kap.4).

\section{Kapitel 6 - Streichung}

Die WHO hat den PF auch „Andere Gesundheitsprobleme“ zugeordnet, die nicht Teil des aktuellen Gesundheitsproblems sind. Diese beziehen sich auf zurückliegende Krankheiten, Gesundheitsstörungen, Verletzungen und Traumata. Sie werden in der vorliegenden Überarbeitung nicht mehr aufgeführt, da ihre Auswirkungen im „Hier und Jetzt“, also im Sinne einer finalen Betrachtungsweise auf unterschiedlichen Ebenen der ICF abgebildet werden können.

\section{Diskussion}

Der Ansatz der DGSMP-Systematik wurde unter Berücksichtigung zwischenzeitlich publizierter Arbeiten, insbesondere der von Geyh et al. [3] und weiterer Rückmeldungen aktualisiert. Unter Beibehaltung der Grundprinzipien wurden die Grundstruktur auf Konsistenz überprüft und neue Aspekte einbezogen. Einige Kategorien wurden eingefügt, gestrichen, zusammengefasst, verschoben sowie redaktionell oder inhaltlich überarbeitet. Zu einer grundlegenden Anpassung kam es nicht, die Grundstruktur der Systematik blieb erhalten.

\section{Der konzeptionelle Zugang der DGSMP zu PF und die Abgrenzung zu anderen ICF-Komponenten}

PF sind in der Person liegende Faktoren, die in Wechselwirkung zu den anderen Komponenten der ICF stehen können. Sie umfassen Gegebenheiten, die nicht Teil des Gesundheitsproblems oder -zustands und nicht Teil der Umwelt sind. Personbezogene Faktoren klassifizieren nicht Personen, sondern sind im Einzelfall bedeutsame Einflussfaktoren einer Person auf deren Funktionsfähigkeit.

Zentral im DGSMP-Ansatz ist die finale Betrachtung der Funktionsfähigkeit als Ausdruck eines Grundprinzips der ICF. Nicht die lebenszeitliche Entwicklung, sondern die Ausprägung zum Beurteilungszeitpunkt wird betrachtet. So wird z. B. nicht der Bildungsprozess, sondern dessen Ergebnis als Bildungsstatus in der ICF erfasst.

Die Betrachtung von PF bezieht sich auf den jeweils aktuellen Zeitpunkt (z. B. Begutachtung) und berücksichtigt in der Regel überdauernde Gegebenheiten. Kurzfristige emotionale Schwankungen werden nicht einbezogen.

In der Vergangenheit wurde kritisiert, dass fast wortgleiche Items in der DGSMP-Systematik und der ICF-Komponente „Körperfunktionen “ die Abgrenzung der Komponenten voneinander erschweren (insbesondere PF von mentalen Funktionen). Gemäß Geyh et al. [3] lassen sich Körperstrukturen, -funktionen und PF auf 
konzeptueller Ebene wie folgt unterscheiden: Körperstrukturen bilden den Akteur (z. B. die Amygdala als das für die Emotionen verantwortliche Hirnareal), Körperfunktionen den Prozess (z. B. emotionale Funktionen) und personbezogene Faktoren das Ergebnis im Sinne der daraus entstehenden Erfahrung (z. B. die Emotion Traurigkeit). Auf klinischer Ebene ergibt sich die Unterscheidung in PF und Körperfunktionen aus der unterschiedlichen Perspektive. Betrachtet man bspw. die Persönlichkeitseigenschaft „Optimismus“, so ist ihre für die Funktionsfähigkeit als Förderfaktor oder Barriere bedeutsame Ausprägung ein PF. Betrachtet man hingegen die Körperfunktion Optimismus, richtet sich der Fokus auf die Schädigung dieser Funktion. Die Körperfunktion bewegt sich vorwiegend im biomedizinischen Modell von Normerwartung und Abweichung.

Die Abgrenzung von PF zur ICF-Komponente „Aktivitäten \& Partizipation“ lässt sich anhand des PF „Gewohnheiten“ nachvollziehen. Aktivitäten beziehen sich auf die Durchführung von Handlungen, während Gewohnheiten im Sinne von PF Verhaltensmuster beschreiben. Nahrung zu sich zu nehmen, ist eine Aktivität. Sich ausschließlich vegetarisch zu ernähren, ist eine Ernährungsgewohnheit und somit ein PF.

Die Abgrenzung von Teilhabe zu PF erschließt sich am Beispiel der Beschäftigung. Der PF beschreibt den Status, bspw. Angestellter sein, während die Teilhabe der gesellschaftlichen Einbindung in eine Bezahlte Tätigkeit entspricht.

Abgrenzungsfragen zwischen PF und Umweltfaktoren zeigen sich insbesondere im Kapitel 5 „Lebenslagen“. Während externe Gegebenheiten Umweltfaktoren darstellen, sind PF in der Person liegende Merkmale. So bezieht sich der PF „Rechtlicher Status“ auf die aus dem Rechtssystem resultierenden Rechte und Pflichten eines Individuums; das Rechtssystem selbst ist ein Umweltfaktor.

\section{Vergleich zwischen DGSMP-Entwurf und der Systematik nach Geyh}

Bei der Revision der DGSMP-Systematik fand eine intensive Auseinandersetzung mit dem Ansatz von Geyh et al. [3] statt. Beiden Ansätzen ist gemeinsam, dass sie generisch sind. Sie bieten eine klare Konzeptualisierung der PF in Abgrenzung zu anderen ICFKomponenten, erlauben eine Gesamtschau auf PF und decken nicht nur Teilaspekte ab. Unterschiede bestehen indes, weil der Ansatz von Geyh et al. auf einem systematischen Review der (insbesondere) psychologischen Literatur sowie ICF-basierter Forschung basiert. Die DGSMP-Systematik wurde primär mittels Expertenkonsensus erarbeitet und berücksichtigt die Forschungsliteratur weniger systematisch. Geyhs Ziel war die Entwicklung eines auf den Klassifikationsprinzipien der WHO basierenden umfassenden und detaillierten internationalen Rahmens zur Entwicklung von Instrumenten für spezifische Anwendungsbereiche. Die DGSMP-Systematik wurde hingegen für den deutschsprachigen Raum mit Fokus auf die (sozialmedizinische) Bedarfsermittlung und Rehabilitation entwickelt.

Auch auf konzeptueller Ebene unterscheiden sich die beiden Ansätze. Die Systematik nach Geyh et al. ist theoriegeleitet. Zudem ist sie psychologisch geprägt und beinhaltet einen großen Anteil an psychologischen PF. Im Gegensatz zur eher praxisorientierten DGSMP-Systematik nehmen bei Geyh et al. Faktoren zur Lebenssituation (z. B. berufliche Situation) und körperliche PF (z. B. kör- perliche Veranlagungen) weniger bis keinen Raum ein. Der Ansatz nach Geyh et al. klassifiziert sowohl stabile Faktoren (Traits wie z. B. übergreifende Verhaltensmuster) als auch kurz- und mittelfristige psychische Zustände (States wie z. B. Gefühle, Motive), während die DGSMP-Systematik aufgrund ihres finalen Ansatzes primär überdauernde PF fokussiert.

Schließlich verwenden Geyh et al. für die Konzeptualisierung der PF einen Lebensspannenansatz, der auch vergangene Erfahrungen berücksichtigt, während die DGSMP-Systematik einen finalen Ansatz verwendet und daher ätiologische Aspekte nicht einbezieht.

\section{Nutzung der Systematik}

Die Systematik soll die Anwender bei der umfassenden Bedarfsermittlung sowie Planung und Durchführung von Teilhabeleistungen unterstützen und sicherstellen, dass alle relevante Aspekte im Zusammenhang mit den Auswirkungen eines Gesundheitsproblems strukturiert berücksichtigt werden. Handlungsleitend ist hierbei, dass PF nur dann zu berücksichtigen sind, wenn sie die Funktionsfähigkeit einer Person beeinflussen.

Vereinzelt vorgetragene grundsätzliche, datenschutzrechtliche Bedenken gegen eine Systematik der PF teilen die Autoren nicht, da mit einer Strukturierung als solcher keine Aussage darüber verbunden ist, welche Faktoren im Einzelfall relevant sind und wie mit diesen verfahren wird. Auch nach Inkrafttreten der Datenschutzgrundverordnung der Europäischen Union und der entsprechenden Umsetzungsgesetze gilt im deutschen Sozialdatenschutzrecht weiterhin: Die Zulässigkeit einer Datenverarbeitung wird danach beurteilt, ob sie im Einzelfall für die Erfüllung einer gesetzlichen Aufgabe erforderlich ist.

Für die Rehabilitation hat der deutsche Gesetzgeber in § 14 Abs. 2 S. 1 SGB IX für alle Leistungsbereiche verbindlich bestimmt, dass im Reha-Prozess eine Bedarfsfeststellung „umfassend“ anhand der Instrumente der Bedarfsermittlung nach § 13 SGB IX zu erfolgen hat. Diese Festschreibung ist gegenüber den für einzelne Leistungsträger geltenden Spezialgesetzen abweichungsfest. Sie ist also als „Aufgabe“ im Sinne des Sozialdatenschutzrechts zu qualifizieren. Die Frage, welche Informationen für die Erfüllung dieser Aufgabe „erforderlich“ sind, ist maßgeblich aus fachlicher Sicht zu bestimmen - selbstverständlich im jeweils gesetzlich vorgegebenen Rahmen. Als eine erste Stufe für die fachliche Umsetzung der gesetzlichen Aufgabe „umfassende Bedarfsermittlung/-feststellung“ haben die Reha-Träger in der Gemeinsamen Empfehlung „RehaProzess“ Grundsätze für Instrumente der Bedarfsermittlung in einer Gemeinsamen Empfehlung konkretisiert (§§ 35-46) [16].

Diese Grundsätze bekräftigen die Bedeutung der ICF und auch der Kontextfaktoren. Die Erhebung von PF im Rahmen der Bedarfsermittlung und -feststellung kann datenschutzrechtlich grundsätzlich als unproblematisch angesehen werden. Die Zulässigkeit der Erhebung und ggf. weiteren Verarbeitung von Kontextfaktoren bestimmt sich allerdings danach, ob sie im Einzelfall zur Erfüllung der gesetzlichen Aufgabe „Bedarfsermittlung und -feststellung“ erforderlich sind. Dies verantwortlich zu beurteilen, obliegt in erster Linie der mit der Aufgabe der Bedarfsermittlung konkret betrauten Person. Sie hat dabei die o.g. Grundsätze für die Bedarfsermittlung und den spezifischen gesetzlichen Auftrag des jeweils verantwortlichen Trägers zu berücksichtigen. 
- Tab. 1 Struktur der Systematik von Geyh et al. aus 2019 [3].

\begin{tabular}{|c|l|l|l|l|}
\hline Kapitel & \multicolumn{3}{|c|}{ Anzahl Kategorien } \\
\hline Individuelle Fakten & Gesamt & 2. Ebene & 3. Ebene & \\
\hline i1 Soziodemographische Charakteristika & & & \\
\hline i2 Position im unmittelbaren sozialen Kontext & 11 & 8 & 3 & \\
\hline i3 Persönliche Lebensgeschichte und Biographie & 7 & 5 & 2 & \\
\hline Subjektives Erleben & & 2 & & \\
\hline i4 Gefühle & 10 & & 8 \\
\hline i5 Gedanken und Überzeugungen & 83 & 51 & \\
\hline i6 Motive & 37 & 3 & 18 & 24 \\
\hline Wiederkehrende Muster des Verhaltens und Erlebens & & & 16 \\
\hline i7 Übergreifende Verhaltens- und Erlebensmuster & 17 & 4 & 8 & \\
\hline Gesamt & 167 & 32 & 90 \\
\hline
\end{tabular}

- Tab. 2 Struktur DGSMP-Systematik von 2010.

\begin{tabular}{|c|c|c|c|c|}
\hline \multirow[t]{2}{*}{ Kapitel } & \multicolumn{4}{|c|}{ Anzahl Kategorien } \\
\hline & Gesamt & 2. Ebene & 3. Ebene & 4. Ebene \\
\hline i1 Allgemeine Merkmale einer Person & 9 & 3 & 6 & \\
\hline i2 Physische Faktoren & 9 & 2 & 7 & \\
\hline i3 Mentale Faktoren & 10 & 10 & & \\
\hline i4 Einstellungen, Grundkompetenzen und Verhaltensgewohnheiten & 20 & 20 & & \\
\hline i5 Lebenslage und sozioökonomische/-kulturelle Faktoren & 9 & 9 & & \\
\hline i6 Andere Gesundheitsfaktoren & 2 & 2 & & \\
\hline Gesamt & 59 & 46 & 13 & \\
\hline
\end{tabular}

- Tab. 3 Struktur DGSMP-Systematik von 2019.

\begin{tabular}{|l|c|c|c|c|}
\hline Kapitel & \multicolumn{3}{|c|}{ Anzahl Kategorien } \\
\hline & Gesamt & 2. Ebene & 3. Ebene & 4. Ebene \\
\hline i1 Allgemeine Merkmale einer Person & 5 & 5 & & \\
\hline i2 Physische Faktoren & 5 & 5 & & \\
\hline i3 Mentale Faktoren & 10 & 10 & & \\
\hline i4 Einstellungen, Grundkompetenzen und Verhaltensgewohnheiten & 25 & 25 & & \\
\hline i5 Lebenslage und sozioökonomische/-kulturelle Faktoren & 10 & 10 & & \\
\hline Gesamt & 55 & 55 & & \\
\hline
\end{tabular}

> Tab. 4 Veränderungen in der Systematik 2010 zu 2019.

\begin{tabular}{|l|c|c|c|}
\hline Anzahl: & $\mathbf{2 0 1 0}$ & $\mathbf{2 0 1 9}$ & \\
\hline Kapitel & 6 & 5 & \\
\hline Blöcke & 7 & 7 & \\
\hline Kategorien & 59 & 55 & \\
\hline Veränderungen von 2010 zu 2019 & & Anzahl & betroffene Items/Kategorien \\
\hline Kategorien neu & & 13 & $i 215, i 225, i 230, i 411, i 418, i 420, i 421, i 431, i 436, i 438, i 460, i 513, i 527$ \\
\hline Kategorien gestrichen & & 5 & $i 130, i 439, i 459, i 610, i 615$ \\
\hline Bezeichnung der Kategorien geändert & & 10 & $i 122, i 130, i 210, i 220, i 320, i 453, i, 510, i 525, i 530, i 550$ \\
\hline
\end{tabular}




\section{FAZIT UND AUSBLICK}

Die überarbeitete DGSMP-Systematik der Personbezogenen Faktoren wird gestützt durch die verstärkte Verankerung der ICF und des ihr zugrundeliegenden bio-psycho-sozialen Modells im neuen trägerübergreifenden Recht des SGB IX. Diese Systematik soll es Nutzern ermöglichen, relevante Einflüsse aus dem Lebenshintergrund einer Person strukturiert zu beschreiben und zu dokumentieren.

Mit ihr können Einflüsse auf die Teilhabe eines Menschen auf Grundlage des bio-psycho-sozialen Modells umfassend und transparent dargestellt werden. Dem Betroffenen selbst wird damit der Weg zu einer justiziablen Leistungsallokation erleichtert. Insbesondere in der Sozialmedizin, im Rehabilitationswesen sowie im (sozial-)rechtlichen Kontext (SGB IX; UN-BRK) ist dieses Vorgehen anerkannt und Grundlage für das Handeln der jeweiligen Akteure.

Die Anwendbarkeit und der Nutzen der vorliegenden Systematik sind in den nächsten Jahren mittels Validierungsstudien im Kontext der Bedarfsermittlung und in Rehabilitations- und Eingliederungsprozessen zu evaluieren.

\section{Interessenkonflikt}

Die Autoren geben an, dass kein Interessenkonflikt besteht.

Literatur

[1] Deutsches Institut für Medizinische Dokumentation und Information (DIMDI), Hrsg. Internationale Klassifikation der Funktionsfähigkeit, Behinderung und Gesundheit, ICF. Köln: Deutscher Ärzte-Verlag; 2005

[2] Schwegler U, Peter C, Trezzini B et al. Toward transparent documentation in medical work capacity evaluations: identifying personal factors in medical reports on Swiss disability claimants with chronic widespread pain. Int J Rehabil Res 2013; 36: 298-307

[3] Geyh S, Schwegler U, Peter C et al. Representing and organizing information to describe the lived experience of health from a personal factors perspective in the light of the International Classification of Functioning, Disability and Health (ICF): a discussion paper. Disabil Rehabil 2019; 41: 1727-1738

[4] Schwegler U, ICF und ressourcenorientierte Abklärung der Arbeitsfähigkeit. In: Riemer-Kafka G, Hürzeler M, Hrsg. Das indikatorenorientierte Abklärungsverfahren. Luzerner Beiträge zur Rechtswissenschaft. 119: Zürich: Stämpfli; 2018: 1-29
[5] Stephen D, Gianopoulos I, Kerr P. Determination and Classification of the Problems Experienced by Hearing-impaired Elderly People. Audiology 2001; 40: 294-300

[6] Ueda S, Okawa Y. The subjective dimension of functioning and disability: what is it and what is it for? Disabil Rehabil 2003; 25 : 596-601

[7] Heerkens Y, Engels ], Kniper C et al. The Use of the ICF to describe work related factors influencing the health of employees. Disability \& Rehabilitation 2004; 26: 1060-1066

[8] Badley Elizabeth M: More than facilitators and barriers: Fitting the full range of environmental and personal contextual factors into the ICF model; 12th Annual North American Collaborating Center - Conference on ICF. Vancouver (Canada) 5.-7. Juni 2006. Im Internet: www. secure.cihi.ca/cihiweb/en/downloads/Elizabeth \%20Badley \%20-\%20 Looking\%20Ahead.pdf 26.01.2011

[9] Viol M, Grotkamp S, van Treek B et al. Personbezogene Kontextfaktoren, Teil I. Gesundheitswesen 2006; 68: 747-759

[10] Howe T]. The ICF Contextual Factors related to speech language pathology. Internat Journal of Speech-Language Pathology 2008; 10 : 27-37

[11] $\mathrm{Ng} \mathrm{L}$, Khan F. Identification of personal factors in motor neurone disease: a pilot study. Rehabil Res Pract 2011, doi:10.1155/2011/871237

[12] Salvador-Carulla L, Walsh CO, Alonso F et al. eVITAL: A preliminary taxonomy and electronic toolkit of health-related habits and lifestyle. Sci World J doi:10.1100/2012/379752

[13] Grotkamp S, Cibis W, Behrens ] et al. PF der ICF - Entwurf der AG ICF des Fachbereichs II der Deutschen Gesellschaft für Sozialmedizin und Prävention (DGSMP). Gesundheitswesen 2010; 72: 908-916

[14] Grotkamp S, Cibis W, Nüchtern E et al. Personbezogene Faktoren der ICF - Beispiele zum Entwurf der AG „ICF“ des Fachbereichs II der Deutschen Gesellschaft für Sozialmedizin und Prävention (DGSMP). Gesundheitswesen 2012; 74: 449-458

[15] Grotkamp S, Cibis W, Nüchtern E et al. Personal Factors in the International Classification of Functioning, Disability and Health: Prospective Evidence. Austral ] Rehabil Counsel 2012; 18: 1-24

[16] Bundesarbeitsgemeinschaft für Rehabilitation e.V.(BAR). Im Internet: www.bar-frankfurt.de/ 05.08.2019

[17] Rapp RS, Queri S. Validierung von Personbezogenen Faktoren im Bereich der onkologischen Brustkrebsrehabilitation - eine DelphiBefragung auf nationaler Ebene. Gesundheitswesen 2017; 79: 96-104

[18] Mahdi S, Ronzano N, Knüppel A et al. An international clinical study of ability and disability in ADHD using the WHO-ICF framework. European Child \& Adolescent Psychiatry doi:10.1007/s00787-0181124-1

[19] Schreier M. Qualitative content analysis in practice. London: Sage; 2012

[20] Deiglmayr A, Schalk L, Stern E. Begabung, Intelligenz, Talent, Wissen, Kompetenz und Expertise: eine Begriffsklärung. In: U. Trautwein \& M. Hasselhorn: Begabungen und Talente. Göttingen: Hogrefe 2017: 1-16

[21] Goldberg L. The development of markers for the Big-Five factor structure. Psychological Assessment 1992; 4: 26-42 